\title{
Localization of the glueball and possible decuplets of tensor mesons
}

\author{
Michał Majewski* \\ Department of Theoretical Physics II, University of Lodz \\ Pomorska 149/153, 90-236 Lodz, Poland
}

June 23, 2021

\begin{abstract}
We continue the study of flavor aspects of strong interaction. The answer to the question of glueball existence is probably a necessary step in this investigation. The existence of the glueball implies the existence of the decuplet. The model we consider requires that the mass of meson decuplet dominated by the glueball is enclosed between the masses of the ideal nonet $\mathrm{N}$ and $\mathrm{S}$ states.

This description is applied to the two sets of the tensor mesons. One of them comprise the well known particles which are attributed to the widely known nonet $2^{++}$. We argue that they belong to a decuplet if there exists the isoscalar tensor meson having the mass obeying the condition on glueball dominated meson; the meson $f_{2}(1430)$ could be a candidate. Another set includes large number of mesons lying in the $2 \mathrm{GeV}$ region. This set is used to test ability of the model to select particles belonging to the decuplet.
\end{abstract}

\section{Introduction}

The quark-gluon picture of strong interactions and the glueball hypothesis 1 posed a question of experimental confirmation of the glueball existence. This requires, first of all, identifying the isoscalar meson which is not a $q \bar{q}$ state. The most direct way to achieve this would be the discovery of an isoscalar meson with exotic signature $J^{P C}$, i.e. having such combination of J,P,C quantum numbers which is not allowed for the $q \bar{q}$ states. The exotic isoscalar meson would be the pure glueball state as it cannot mix with the isoscalar $q \bar{q}$. The exotic glueballs are admitted by some models like constituent glue or lattice QCD but were not observed so far. It is thus not possible to discover the glueball by studying the properties of a single particle.

There exist, however, a large number of nonexotic isoscalar mesons attributed to various signatures $J^{P C}$. The non-exotic glueballs may be hidden among them. We expect the glueball to be the one of three isoscalar unphysical states belonging to the same multiplet. Its mixing with $q \bar{q}$ isoscalar nonet states enlarges the multiplet of the light mesons to a decuplet. In order to extract the

*e-mail: m.majewski@merlin.phys.uni. lodz.pl 
glueball state $(\mathrm{G})$ from the measurable physical isoscalar states we must know mixing matrix (MM) of the decuplet. The MM is a quantity of great importance for $\mathrm{G}$ search and to construct it accurate data and reliable procedure are required.

The $q \bar{q}$ states and the glueball are related due to the mixing. The pure $q \bar{q}$ states reflect nonet properties of the mesons. When glueball gets mixed with the $q \bar{q}$ states, one should expect modification of the nonet properties. Conversely: specific properties of some observed nonet may suggest the influence of glueball mixing. Many nonets can be regarded as more or less deformed. Two of them: $0^{-+}\left(\pi, K, \eta, \eta^{\prime}\right)$ and $2^{++}\left(a_{2}, K_{2}^{*}, f_{2}, f_{2}^{\prime}\right)$ were once considered as the most promising in this respect (the former has bizarre mixing angle; the latter suffers with too large difference between $a_{2}$ and $f_{2}$ masses).

Particular interest just in these two nonets was induced by observations of the $\iota(1440)$ and $\Theta(1640)$ signals in the $J / \Psi$ radiative decays (we use original symbols). To these signals there were attributed the $0^{-+}$and $2^{++}$signatures, respectively. There was a hope that establishing the connection between the meson and the deformation of the nonet will confirm the existence of the glueball and, at the same time, explain the deformation mechanism [2]. These attempts failed for several reasons (we comment on this later). The failures suggest more scrupulous examination of the procedure applied and improving or replacing it by a more effective one. However, the original idea about glueball mixing should be preserved. Thus, for revealing the non-exotic glueball we have to investigate ten related mesons which form a decuplet. Yet this should not be regarded an obstacle. The analysis of the mixing is the necessity in meson spectroscopy even if the glueballs would be already discovered.

\section{Standard diagonalization of the mass operator}

To be more specific, we examine how the unphysical isoscalar states $(q \bar{q})_{\text {octet }}$, $(q \bar{q})_{\text {singlet }}$ and $G$ are distributed among the states of the isoscalar physical mesons $x_{1}, x_{2}, x_{3}$. The MM transforms these states from the basis where their properties are defined to the basis where the mass operator is diagonal. The way to determine MM is diagonalization of the initial mass operator. We use quadratic mass operator; the particle symbol means its name or mass squared.

The most obvious procedure is standard diagonalization. In the simplest case the $2 \times 2$ matrix describing mixing of two nonet states is diagonalized.

$$
\left[\begin{array}{cc}
x_{8} & \alpha \\
\alpha & x_{0}
\end{array}\right] \stackrel{\operatorname{diag}}{\longrightarrow}\left[\begin{array}{cc}
x_{1} & 0 \\
0 & x_{2}
\end{array}\right]
$$

Here $x_{8}$ is an octet isoscalar meson, $x_{0}$ is a $\mathrm{SU}(3)$ singlet, $\alpha$ is a mixing parameter, $x_{1}, x_{2}$ are the isoscalar physical mesons. The mass of the octet isoscalar meson $x_{8}$ is assumed to be determined by (quadratic) Gell-Mann - Okubo (GMO) mass formula:

$$
x_{8}=\frac{1}{3} a+\frac{2}{3} b
$$

where $a$ is an isovector meson; $b$ (a parameter having sense of unphysical $s \bar{s}$ state mass squared) is given by

$$
b=2 K-a
$$


$K$ is the strange meson.

To diagonalize the matrix we must know its elements. In the relation (1) two of them $-x_{0}$ and $\alpha$ are always unknown but they can be eliminated if the masses of $x_{1}$ and $x_{2}$ mesons are known. This can be done by using the invariants of diagonalizing transformation: $\operatorname{tr}\left(m^{2}\right)$ and $\operatorname{det}\left(m^{2}\right)$. For $2 x 2$ matrix the invariants are very simple:

$$
\begin{aligned}
& x_{8}+x_{0}=x_{1}+x_{2} \\
& x_{8} x_{0}-\alpha^{2}=x_{1} x_{2}
\end{aligned}
$$

Therefore, if the masses of physical mesons are known, no further information is necessary: the procedure of diagonalization is model independent.

The situation changes when three isoscalar states are mixed. We want to diagonalize $3 \times 3$ symmetric matrix

$$
\left[\begin{array}{ccc}
x_{8} & \alpha & \beta \\
& x_{0} & \gamma \\
& & G
\end{array}\right] \stackrel{\operatorname{diag}}{\longrightarrow}\left[\begin{array}{ccc}
x_{1} & 0 & 0 \\
& x_{2} & 0 \\
& & x_{3}
\end{array}\right] .
$$

Using (2) for $x_{8}$ and assuming that the physical mesons $x_{1}, x_{2}, x_{3}$ are known, we find that the procedure (6) depends on 5 unknown parameters. These parameters are related to three invariant functions of the diagonalizing transformation: $\operatorname{tr}\left(m^{2}\right), \operatorname{tr}\left(\left(m^{2}\right)\right)^{2}$ and $\operatorname{det}\left(m^{2}\right)$. Therefore, two of the unknown parameters cannot be eliminated. If we want to diagonalize the matrix $m^{2}$, we must fix them. For this purpose one usually resorts to the approximations simplifying the mass operator. It is convenient to make the approximations in the ideal quark-glueball basis

$$
N=\frac{u \bar{u}+d \bar{d}}{\sqrt{2}}, \quad S=s \bar{s}, \quad G
$$

which helps in better understanding the physical sense of the made approximations. A number of such simplified operators constructed with various motivations (or being the response to various demands) have been proposed (see e.g. 3 ); These operators are different and imply different MM's.

Unfortunately, the above approach did not appear to be reliable. There were many attempts to describe mixing of the $\Theta$ meson with $2^{++}$nonet (see e.g. [2]) and the $\iota$ meson with the $0^{-+}$nonet [5], but no definite answer has been obtained to this question. It became finally clear that the signature $J^{P C}=$ $0^{++}$(not $2^{++}$!) should be attributed to the signal $\Theta$. Also it appears that in the neighbourhood of the $\iota$ meson there exist several pseudoscalar mesons constituting a separate multiplet. So in both these cases the mixing partners have been selected in a wrong way. We see that such an approach may lead to accidental result which cannot be treated as reliable prediction. The failure of these attempts have slow down the progress in studies of meson spectroscopy. It is thus desirable to have a procedure which could help to avoid similar confusions even if only partly.

There were also attempts to determine the mass of a pure glueball (see e.g. [6]). The most prominent are calculations of lattice QCD [7] predicting the glueball masses for different $J^{P C}$. The lowest predicted states are

$$
0^{++}(1710), \quad 0^{-+}(2560), \quad 2^{++}(2300) \text {. }
$$

Their masses much exceed the expected values. 


\section{Master equations for light meson multiplets}

\subsection{Conditions allowing multiplet existence}

There exists another model well describing the properties of known octet and nonets of $S U(3)$ broken symmetry which provides as well the very convenient tool for investigating the decuplets. We call it the model of vanishing exotic commutators (VEC) 11 (see Appendix 1.); below, the term "multiplet" means the multiplet of VEC-broken $S U(3)$ symmetry.

VEC is based on the postulate of vanishing of the sequence of exotic commutators build out of generators and their time derivatives. This sequence can be transformed [8, 9] into a sequence of algebraic equations describing meson multiplets called the master equations (ME)

$$
\sum_{i} l_{i}^{2} x_{i}^{r}=\frac{1}{3} a^{r}+\frac{2}{3} b^{r}, \quad r=0,1,2, \ldots
$$

where $r$ is the power index; $a, b, K, x_{i}$ have been already described and the index $i$ runs over all isoscalar mesons of the multiplet. The number of the isoscalar meson is growing with growing mass:

$$
x_{i}<x_{i+1}
$$

The coefficients $l_{i}$ express octet contents of the isoscalar states $x_{i}$

$$
\left|x_{8}\right\rangle=\sum_{i} l_{i}\left|x_{i}\right\rangle
$$

where $l_{i}$ are real numbers because the wave functions $x_{8}$ and $x_{i}$ describe uncharged particles. Hence,

$$
l_{i}^{2} \geq 0 \quad i=1,2, \ldots
$$

This is the requirement that the octet contents $l_{i}^{2}$ of the physical isoscalar states $x_{i}$ should be positive.

The ME (9) is a sequence of equations which are linear with respect to unknown variables $l_{i}^{2}$. The solution is determined by the masses of octet states $a, b$ which are indicated by experiment and the masses of undefined number of physical isoscalar mesons $x_{i}$. As the $a, b$ are assumed to be known from experiment they are considered as diagonal elements of the multiplet (they may be the same for different multiplets, e.g. nonet and decuplet); $x_{i}$ result from diagonalization of its isoscalar sector.

The functions $l_{i}^{2}$ being the solution of the ME, are not a priori positive; therefore we require them to satisfy the conditions (12). These conditions restrict the masses of particles belonging to the multiplet; the particles may form multiplet only if their masses satisfy these conditions.

\subsection{Number of master equations, solvability conditions and genesis of the multiplet mass formulae}

The number of equations (9) which are to be taken into account is not declared in advance because it depends on the multiplet. Yet this number cannot be

\footnotetext{
${ }^{1}$ formerly named the exotic commutator (ECM) model
} 
smaller than the number of variables $l_{i}^{2}$ since we want to describe the multiplet. So they form the minimal (basic) sequence of ME describing the multiplet.

The multiplet can also be described by the system of ME larger than the basic one. Such a system for linear equations is overdetermined. Hence, to be a solution the $l_{i}^{2}$ 's must satisfy some extra solvability conditions. The $l_{i}^{2}$ 's depend only on the masses of the multiplet particles, therefore the extra condition can be satisfied only at cost of the mass restriction.

The solvability conditions are described by the sequence (9) as well. Inserting the solution of the basic sequence of $\mathrm{ME} l_{i}^{2}$ into any of the subsequent equations we obtain some relation $F_{j}=0$ which depends only on the masses of the multiplet. Hence, it constitutes a MF. We thus get a set of MF's

$$
F_{j}=0, \quad j=1,2,3 \ldots
$$

Each of the MF's corresponds to one of the extra equations (9) and puts on the masses one restriction. As the multiplet has finite number of particles the number of independent relations $F_{j}=0$ complying with data must be finite. The sequence of equations describing the multiplet ends when the subsequent $F_{k}=0$ contradicts the data.

Note that the MF is not necessary for existence of the multiplet. On the other hand, the multiplet may have more than one MF. Since the number of MF is finite we can number them. If $F_{1}=0$ is satisfied by the data, the multiplet has at least one MF. Then we should check whether the second MF $\left(F_{2}=0\right)$ is also satisfied. The multiplet has two MF's if the latter equation complies with the data and does not reduce to the previous restriction. Clearly we can prolong this procedure and check the existence of the $F_{3}=0$ and so on.

However, it may also happen that starting from some $F_{k}=0$, the following MF's comply with data but reduce to the earlier result. Then the corresponding equations (9) do not influent the description of the multiplet and may be abandoned. Hence, also in this case the sequence (9) describing the multiplet is finite.

Thus the number of ME (9) describing the multiplet is equal to sum of two numbers: the number of isoscalar states and the number of MF's relating the masses of the multiplet.

The multiplets having different number of MF's are described by VEC as different multiplets. They have the same basic sequence of ME but they are described by different number of equations (9).

We can see that the VEC model offers the description of wide variety of the multiplets. The study on existence the multiplets as well as the mechanisms of their appearance is subject of the meson spectroscopy. The VEC model is a promising tool for investigating these phenomena.

The features of the VEC description can be apparently seen on example of the nonets. There are three allowed kinds of the nonet which correspond to zero, one and two MF's obeyed by them. The procedure defining the kinds of nonet has been already presented [8, 11] for other purpose. Now we remind it stressing the aspects which will be useful during investigating the decuplet.

\subsection{Description of the nonets}

The basic (minimal) sequence for the description of nonet consists of the first two equations (9). Depending on the number of MF's three types of the nonets 
are distinguished which are named: the Gell-Mann - Okubo (GMO), Schwinger (S) and Ideal (I) for the cases of 0, 1 and 2 MF's, respectively.

- Gell-Mann - Okubo (GMO) nonet

This type of the nonet arises as a solution of the system of the first two ME (9). In this case we have no MF. The solution is

$$
\begin{aligned}
& l_{1}^{2}=\frac{x_{2}-x_{8}}{x_{2}-x_{1}} \\
& l_{2}^{2}=\frac{x_{8}-x_{1}}{x_{2}-x_{1}}
\end{aligned}
$$

or

$$
\begin{aligned}
& l_{1}^{2}=\frac{1}{3} \frac{\left(x_{2}-a\right)+2\left(x_{2}-b\right)}{x_{2}-x_{1}}, \\
& l_{2}^{2}=\frac{1}{3} \frac{\left(a-x_{1}\right)+2\left(b-x_{1}\right)}{x_{2}-x_{1}} ;
\end{aligned}
$$

The nonet is described by the mixing angle $\vartheta$ as a parameter. The angle $\vartheta$ is defined as

$$
\tan ^{2} \vartheta=\frac{l_{1}^{2}}{l_{2}^{2}} .
$$

The solution (13) of ME is determined by the masses. However, not all possible solutions describe the nonet. The masses of the isoscalar mesons are constrained by the relations

$$
x_{1}<x_{8}<x_{2}
$$

which follow from the conditions (12); here $x_{8}$ is GMO mass (2). The GMO nonet describes pseudoscalar mesons: $\pi, K, \eta, \eta^{\prime}$.

- Schwinger (S) nonet

This type of nonet arises by solving the system of three ME 9

Both the solution (14) and the definition (15) remain true, but now the masses are related by the MF

$$
\left(a-x_{1}\right)\left(a-x_{2}\right)+2\left(b-x_{1}\right)\left(b-x_{2}\right)=0
$$

which is called the Schwinger MF.

The constraints on the masses of the S-nonet are stronger than on the GMO-nonet ones. It follows from (14) and (17) that the masses of Snonet must comply with one of two possible mass ordering rules (MOR) [10]:

$$
\begin{aligned}
& x_{1}<a<x_{2}<b, \quad \tan ^{2} \vartheta<\frac{1}{2}, \\
& a<x_{1}<b<x_{2}, \quad \tan ^{2} \vartheta>\frac{1}{2} .
\end{aligned}
$$

This solution describes most of the nonets independently of their $J^{P C}$. 
- Ideal (I) nonet

The third type of nonet arises as the solution of the four ME (9).

Apart from (17) also the second MF emerges

$$
a\left(a-x_{1}\right)\left(a-x_{2}\right)+2 b\left(b-x_{1}\right)\left(b-x_{2}\right)=0 .
$$

Solving (17) and (19) with respect to $x_{1}$ and $x_{2}$ we find

$$
x_{1}=a, \quad x_{2}=b, \quad l_{1}^{2}=\frac{1}{3}, \quad l_{2}^{2}=\frac{2}{3}, \quad \tan ^{2} \vartheta=\frac{1}{2} .
$$

Hence, the nonet of the third type is ideal. None of the observed meson nonets is strictly I-nonet but many of them deviate from it only slightly. Perhaps the most ideal are nonets $1^{--}$and $3^{--}$.

Further increasing of the number of equations (9) does not change the solution (20). The S-nonet has one MF; the I-nonet has two MF's. Their mixing angles are different.

Notice that the equations (17) and (19) have also the solution $x_{1}=b$ and $x_{2}=a$ 8. This solution does not describe any known multiplet. For choosing the physical solution describing the I-nonet we need to know which of the $x_{i}$ mesons is $\mathrm{N}$ (or S) state (7). The old choice of the solution (20) was just the result of common consent that the heavier of isoscalar mesons is the hidden strange $S=s \bar{s}$ state.

The same concerns the Schwinger nonet but this time we must know which of the observed $x_{i}$ mesons is dominated (not completely determined) by $\mathrm{N}$ (or S) state.

The N/S domination patterns of the isoscalar states is identical for all nonets independently of their signature $J^{P C}$ and the number of MF's satisfied by them.

The notion of dominance pattern will play important role in our investigation of the decuplet.

\section{Description of decuplets}

\subsection{Diagonalization and wave functions}

The basic sequence describing the decuplet consists of three equations (9). There are two possible kinds of the decuplet: the one having no MF and the one having one MF. The functions $l_{i}^{2}$ solving the basic sequence of ME (9) are explicitly expressed in terms of the masses

$$
\begin{aligned}
& l_{1}^{2}=\frac{1}{3} \frac{\left(x_{2}-a\right)\left(x_{3}-a\right)+2\left(x_{2}-b\right)\left(x_{3}-b\right)}{\left(x_{1}-x_{2}\right)\left(x_{1}-x_{3}\right)}, \\
& l_{2}^{2}=\frac{1}{3} \frac{\left(x_{1}-a\right)\left(x_{3}-a\right)+2\left(x_{1}-b\right)\left(x_{3}-b\right)}{\left(x_{2}-x_{1}\right)\left(x_{2}-x_{3}\right)}, \\
& l_{3}^{2}=\frac{1}{3} \frac{\left(x_{1}-a\right)\left(x_{2}-a\right)+2\left(x_{1}-b\right)\left(x_{2}-b\right)}{\left(x_{3}-x_{1}\right)\left(x_{3}-x_{2}\right)} .
\end{aligned}
$$

From the two kinds of the decuplets the more reliable and easier to complete is the one having MF. We restrict our attention to it. 
The MF is 11, 12,

$$
\left(x_{1}-a\right)\left(x_{2}-a\right)\left(x_{3}-a\right)+2\left(x_{1}-b\right)\left(x_{2}-b\right)\left(x_{3}-b\right)=0 .
$$

The conditions (12) and MF (22) impose a number of constraints on the masses. These constraints can be presented in the form of the mass ordering rule (MOR) (see Appendix 2.)

$$
x_{1}<a<x_{2}<b<x_{3} .
$$

This rule is an indispensable tool for selecting the candidates for decuplet.

Let us introduce the mixing matrix $\mathrm{U}$ transforming isoscalar states of exact symmetry $S U(3)$ into the physical ones:

$$
\left[\begin{array}{l}
x_{1} \\
x_{2} \\
x_{3}
\end{array}\right]=U\left[\begin{array}{l}
x_{8} \\
x_{0} \\
G
\end{array}\right]
$$

the initial and final states have been described in the section 2. This matrix is orthogonal and can be written in the form

$$
U=\left[\begin{array}{ccc}
c_{1} & -s_{1} c_{2} & s_{1} s_{2} \\
s_{1} c_{3} & c_{1} c_{2} c_{3}-s_{2} s_{3} & -c_{1} s_{2} c_{3}-c_{2} s_{3} \\
s_{1} s_{3} & c_{1} c_{2} s_{3}+s_{2} c_{3} & -c_{1} s_{2} s_{3}+c_{2} c_{3}
\end{array}\right],
$$

where $c_{j}=\cos \vartheta_{j}, s_{j}=\sin \vartheta_{j},(j=1,2,3)$ and $\vartheta_{j}$ are Euler angles: $0 \leq \vartheta_{1}<\pi ; \quad 0 \leq\left(\vartheta_{2}, \quad \vartheta_{3}\right)<2 \pi$.

The elements of the first column are just the coefficients $l_{1}, l_{2}, l_{3}$ introduced in (11). Their squares are the solution (21) of the ME. Therefore, we have:

$$
c_{1}=l_{1} ; s_{1} c_{3}=l_{2} ; \quad s_{1} s_{3}=l_{3} .
$$

Hence, the absolute values of the trigonometric functions of the Euler angles $\vartheta_{1}$ and $\vartheta_{3}$ are expressed in terms of masses.

To compare the predictions with data we express the MM in the basis of the $\mathrm{N}, \mathrm{S}, \mathrm{G}$ states. In this basis the physical isoscalar states are described by the matrix $\mathrm{V}$

$$
\left[\begin{array}{l}
x_{1} \\
x_{2} \\
x_{3}
\end{array}\right]=V\left[\begin{array}{l}
N \\
S \\
G
\end{array}\right]
$$

where

$$
V=U Q
$$

and the matrix $\mathrm{Q}$

$$
Q=\left[\begin{array}{ccc}
\frac{1}{\sqrt{3}} & -\sqrt{\frac{2}{3}} & 0 \\
\sqrt{\frac{2}{3}} & \frac{1}{\sqrt{3}} & 0 \\
0 & 0 & 1
\end{array}\right],
$$

transforms the bases

$$
\left[\begin{array}{c}
x_{8} \\
x_{0} \\
G
\end{array}\right]=Q\left[\begin{array}{c}
N \\
S \\
G
\end{array}\right] .
$$

The relations (21), (25) and (26) show that the matrix $\mathrm{V}$ depends on trhe masses of decuplet particles. However, the angle $\vartheta_{2}$ remains unknown. It cannot 
be determined on the basis of the solution of ME and is a free parameter of the MM. This freedom can be used for giving $\mathrm{V}$ some desirable specific feature. In the case of $\mathrm{G}$ mixing we require its flavor independence:

$$
<G\left|m^{2}\right| N>=\sqrt{2}<G\left|m^{2}\right| S>
$$

which implies

$$
\tan \vartheta_{2}=\frac{c_{3} s_{3}}{c_{1}} \frac{x_{3}-x_{2}}{\left(x_{3}-x_{1}\right)-\left(x_{3}-x_{2}\right) c_{3}^{2}} .
$$

With this value of $\vartheta_{2}$ the MM depends only on the masses. We call it the glueball shaped MM and label as $V_{G}$. Its explicit form is:

$$
\begin{aligned}
& V_{G}= \\
& {\left[\begin{array}{ccc}
\frac{1}{\sqrt{3}} c_{1}-\sqrt{\frac{2}{3}} s_{1} c_{2} & -\sqrt{\frac{2}{3}} c_{1}-\frac{1}{\sqrt{3}} s_{1} c_{2} & s_{1} s_{2} \\
\frac{1}{\sqrt{3}} s_{1} c_{3}+\sqrt{\frac{2}{3}}\left(c_{1} c_{2} c_{3}-s_{2} s_{3}\right) & -\sqrt{\frac{2}{3}} s_{1} c_{3}+\frac{1}{\sqrt{3}}\left(c_{1} c_{2} c_{3}-s_{2} s_{3}\right) & -c_{1} s_{2} c_{3}-c_{2} s_{3} \\
\frac{1}{\sqrt{3}} s_{1} s_{3}+\sqrt{\frac{2}{3}}\left(c_{1} c_{2} s_{3}+s_{2} c_{3}\right) & -\sqrt{\frac{2}{3}} s_{1} s_{3}+\frac{1}{\sqrt{3}}\left(c_{1} c_{2} s_{3}+s_{2} c_{3}\right) & -c_{1} s_{2} s_{3}+c_{2} c_{3}
\end{array}\right]}
\end{aligned}
$$

To have this matrix completely determined we must fix all signs of the trigonometric functions $c_{i}, s_{i}(\mathrm{i}=1,2,3)$ 11. For some of them the sign is arbitrary due to the sign ambiguity of the matrix $\mathrm{V}$ but not for all. The other signs can be determined if N-,S-,G- domination is known for two of the three $x_{i}$ states - It turns out that the information about the domination has much stronger impact on the shape of the MM than the particular numerical values of the functions $c_{i}, s_{i}$. The former determine the character of the MM while the latter only change the numerical values of its elements. Therefore, it is important to examine the possible types of dominations and find out their implications and origin.

\subsection{G-, N-, S- domination pattern of the decuplet and the localization of glueball}

The genuine (not degenerated) states of the decuplet $x_{i}$ mesons are superpositions of three unphysical isoscalar states: $x_{8}, x_{0}, \mathrm{G}$. But it is more convenient to express the states $x_{i}$ as linear combinations of N, S, G ones. The combinations must be different because the $x_{i}$ particles are different. Yet to be noticeable different, each isoscalar meson must be clearly dominated by one of the basis states. We need the information which of the isoscalar mesons $x_{i}$ is dominated by $\mathrm{G}$. This information is not always available directly from experiment 2 . If this is the case we can try to make use of the properties of the remaining $x_{i}$ mesons (the normalization of their amplitudes) to infer which meson is dominated by $\mathrm{G}$. We now explain how this can be done; we start by reminding the features of the decuplets containing G-state which were indicated by data.

\footnotetext{
${ }^{2}$ The physical meson $x_{i}$ dominated by $\mathrm{G}$ is usually called a "glueball candidate". Observe that G in VEC model is not necessarily the glueball; it may be any scalar of the flavor $\mathrm{SU}(3)$ which is built of other constituents. Identification of the constituents is a separate problem.
} 


\subsubsection{Decuplets including apparent G-dominated state}

So far two decuplet candidates including experimentally recognized G-dominated state have been introduced and discussed [11, 12. They include the $0^{++}$and $0^{-+}$mesons having masses in the interval between $1.2 \mathrm{GeV}$ and $2.4 \mathrm{GeV}$, which is usually considered as room for excited $q \bar{q}$ states. In some cases their existence is not firmly established and many masses are burdened by large errors. In spite of that, gathering these particles into a decuplet is possible due to MOR restrictions on the decuplet masses.

- The $0^{++}$decuplet is composed of the mesons [11]:

$$
a_{0}(1450), \quad K_{0}(1950), \quad f_{0}(1370), \quad f_{0}(1500), \quad f_{0}(2200) / f_{0}(2330) .
$$

Arranging this decuplet has started after it was established that the meson $f_{0}(1500)$ is dominated by $\mathrm{G}$ state [14, 15]. Three of the mesons (34): $a_{0}(1450)$, $f_{0}(1370)$ and $f_{0}(1500)$ belong to "firmly established" particles 14 . One of them is just the $f_{0}(1500)$. This supports the conclusion concerning the existence of the decuplet because, according to $\mathrm{G}-(q \bar{q})$ mixing picture, it can be taken for granted once the existence of non-exotic $\mathrm{G}$ is established.

The enormously large difference between the masses of $K_{0}(1950)$ and $a_{0}(1450)$ mesons may be embarrassing but there are no other candidates. Moreover, the difference between the corresponding states of the lower lying nonet $K_{0}(1430)$ and $a_{0}(980)$ is also large. Therefore, we accept this difference as an experimental fact. Hence, we also accept their very large mass spread (difference between the biggest and the smallest mass of the multiplet). The MF reveals strong correlation between the masses of $K_{0}(1950)$ and $x_{3}$ mesons. The former is measured with large error implying broad dispersion of the predicted $x_{3}$ mass. This is indicated in (34) by double name of the meson $f_{0}(2200) / f_{0}(2330)$. Still larger uncertainty is introduced to the decuplet states by wide experimental dispersion of the $f_{0}(1370)$ mass which, in turn, is correlated with the mass of $a_{0}(1450)$ meson. The present data are not sufficiently accurate to make definite fit but the MOR restrictions considerably reduce the search range.

- The decuplet $0^{-+}$includes the mesons [12]:

$$
\pi(1300), \quad K(1460), \quad \eta(1295), \quad \eta(1405), \quad \eta(1475) .
$$

Its spread is small. According to [14, 16] the meson $\eta(1405)$ is dominated by $\mathrm{G}$. The masses of the isoscalar mesons $x_{i}$ are measured very precisely, but the mass of the $\mathrm{K}(1460)$ is unknown and the error for the $\pi(1300)$ is so big that it also must be assumed unknown. We have at our disposal only one MF to determine these two masses. Moreover, the MF is a cubic equation with respect to each of the masses. Hence, the comprehensible solution can only be approximate. It was found due to MOR restrictions (23). The solving procedure divides the two-dimensional domain of unknowns $a$ and $b$ into four disconnected sub-domains having distinct properties. The solution is looked for in each subdomain separately. In any domain the solution of the ME is dominated by one of the $N, S, G, x_{8}$ states. The type of domination is preserved across each sub-domain. As a solution of the ME we accept the one at the sub-domain which assigns the G-domination to the $x_{2}$ state. The masses of the $\pi(1300)$ and $K(1460)$ mesons belonging to the sub-domain are not precisely determined but the ranges of their variability are of the size of typical experimental error [12]. 


\subsubsection{Any apparent domination and localization of the glueball}

Dominations of the $f_{0}(1500)$ and $\eta(1405)$ mesons by $\mathrm{G}$ were established by experimental observations but not predicted by certain a priori postulated model. The VEC description only reveals that they can be understood as the components of decuplets. The common feature of these two decuplets is that the G dominated isoscalar meson occupies the same central position (of $x_{2}$ ) in the sequence of (of $x_{i}$ ) states. We shall now examine implications of this statement for other decuplets.

The $\mathrm{G}$ domination of the $x_{2}$ meson implies $\mathrm{N}, \mathrm{S}$ domination of the $x_{1}, x_{3}$ mesons, respectively. The G-shaped MM (32) predicts

$$
\left|x_{1}>\sim\right| N>, \quad\left|x_{3}>\sim\right| S>.
$$

This is standard domination pattern of the nonet a, $\mathrm{K}, x_{1}, x_{3}$.

Both nonets and decuplets of mesons are described by the ME (8). The MF of the ideal nonet (17), (19) and the MF of the decuplet (22) are required by consistency condition of the overdetermined system of the first four equations (9). The octet contents $l_{i}^{2}$ ( $\mathrm{i}=1,2$ - for the nonet and $\mathrm{i}=1,2,3$ - for the decuplet) play role of unknown variables of the system; a,b are considered as known constants.

For the I-nonet we have

$$
x_{1}=a, \quad x_{2}=b, \quad\left|x_{1}>=\right| N>, \quad\left|x_{2}>=\right| S>.
$$

The masses of the decuplet mesons should satisfy MF (22). The structures of its isoscalar components $\mid x_{i}>$ are not ideal. However, if the interaction between $\mathrm{G}$ and isoscalar nonet states were switched off (by putting $l_{2}^{2}=0$ ), the ME would describe ideally mixed nonet with $x_{1}, x_{3}$ as isoscalar components

$$
x_{1}^{i d}=a, \quad x_{3}^{i d}=b, \quad\left|x_{1}^{i d}>=\right| N>, \quad\left|x_{3}^{i d}>=\right| S>
$$

and disconnected SU(3)-singlet G. Thus the G-shaped decuplet arises from mixing of the I-nonet with $\mathrm{G}$.

The decuplet mass gaps - the bottom: $x_{1}^{i d}-x_{1}$ and the upper: $x_{3}-x_{3}^{i d}$ arise from mixing. They are known since the decuplet MF is solved. Besides, the gaps depend on spread of the decuplet. Hence, they are different in different decuplets but both for $0^{++}$(where the spread is large) and $0^{-+}$(where it is small) they are much smaller than the I-nonet isoscalar mass difference (38)

$$
x_{1}^{i d}-x_{1}, \quad x_{3}-x_{3}^{i d} \quad<<\left(x_{3}^{i d}-x_{1}^{i d}\right) .
$$

This discloses relative weakness of the G- $(q \bar{q})$ mixing mechanism and explains why converting the nonet into decuplet does not change the original N, S domination assignment of the $x_{1}, x_{3}$ states.

Obviously, the most interesting situation arises when the G-dominated meson among $x_{i}$ is not identified. Then we must first verify whether we are dealing with a decuplet. This can be done by checking MOR restrictions (23). If it is a decuplet complying with (39) then dominations are noticeably. We thus expect for all G-shaped decuplets the same domination pattern

$$
\left|x_{1}>\sim\right| N>, \quad\left|x_{2}>\sim\right| G>, \quad\left|x_{3}>\sim\right| S>.
$$


The mass of the $\mathrm{G}$ dominated state $x_{2}$ is restricted by conditions

$$
a<x_{2}<b
$$

required by MOR (23) and can be found by solving the decuplet MF (22).

\section{Possible decuplets of $2^{++}$mesons}

Our main purpose is to examine the existence of $2^{++}$meson decuplets. The tensor mesons are very curious objects for such investigation because they are numerous with great majority of the isoscalar mesons. Some of them may belong to decuplets. It is a puzzle which kind of the multiplets might be composed of the to date observed signals. Many of the recorded signals need confirmation and most of the listed masses are measured with low accuracy. This concerns first of all the signals descending from the particles having masses close to $2 \mathrm{GeV}$ or above. Therefore, predictions for the higher lying multiplet are tentative and should not be treated too literally. Yet, in spite of that the model brings transparent outline which helps to exploit the scanty, unconfirmed and inaccurate data on individual particles for constructing the possible multiplet.

The earlier studies of the $0^{-+}$and $0^{++}$multiplets [11,12] seemed to suggest that between $\mathrm{G}$ and $q \bar{q}$-excited states there may exist a correlation resembling the chemical affinity [12. It would be interesting to see how such a property could manifest itself in the multiplets of the $2^{++}$mesons.

We begin with the discussion of the best known particles which are usually considered as nonet components.

\subsection{Is the well known tensor nonet really a nonet?}

The currently known nonet of tensor mesons

$$
a_{2}(1320), \quad K_{2}^{*}(1430), \quad f_{2}(1270), \quad f_{2}^{\prime}(1525)
$$

belongs to one of the most early recognized SU(3) multiplets 13. The belief in its nonet status has been lasting many decades (excluding probably a short time of fascination with $\Theta(1640)$ meson). Equally stable - but without any doubt is the belief that the mesons $f_{2}(1270)$ and $f_{2}^{\prime}(1525)$ are almost pure $\mathrm{N}$ and $\mathrm{S}$ states. Perhaps such a stability of opinion concerning this multiplet reflects the fact that subsequent measurements of their masses exhibited only small changes creating no stimulus for reanalysis.

The present analysis was motivated by an attempt to select candidates to higher lying decuplet $2^{++}$, similar to the $0^{++}$and $0^{-+}$ones. This gave rise to examining all signals of tensor mesons. Unexpectedly, among the current data we find the ones which may change multiplet status of the mesons (42).

Two observations are especially important for reanalysis of the multiplet assignment of the tensor mesons (42):

- The masses of the mesons $K_{2}^{* \pm}$ and $K_{2}^{* o}$ are known and individually determined. The measurement is very precise and difference between them much exceeds the experimental errors 14 .

- In few experiments within the region of appearance of the mesons (42) the signals of a mysterious meson $f_{2}(1430)$ were recorded. 
Table 1: Masses of tensor mesons belonging to a possible ground state decuplet. Masses (in $\mathrm{MeV}$ ) and confidence are quoted after [14]

\begin{tabular}{|c|c|c|c|c|c|}
\hline$\bullet a_{2}(1320)$ & $\bullet K_{2}^{* \pm}(1430)$ & $\bullet K_{2}^{* 0}(1430)$ & $\bullet f_{2}(1270)$ & $\bullet f_{2}^{\prime}(1525)$ & $\left.f_{2}(1430)\right)$ \\
\hline $1318.3_{-.6}^{+.5}$ & $1425.6 \pm 1.5$ & $1432.4 \pm 1.3$ & $1275.1 \pm 1.2$ & $1525 \pm 5$ & $\sim 1430$ \\
\hline
\end{tabular}

These observations are not quite new but were abandoned previously because they are silent as long as the mesons (42) are investigated not otherwise than as belonging to a nonet.

The present data on masses and confidence of the mesons (42) and $f_{2}(1430)$ (a likely candidate to the decuplet) are quoted in the tab.1. In other multiplets the difference between masses of charged and neutral $K$-mesons are not measurable or are meaningless but in the case of $K_{2}^{*}$ the difference is significant and cannot be neglected. This raises the question which is unusual for the meson spectroscopy: which of the $K_{2}^{*}$ meson masses describes the breaking of $\mathrm{SU}(3)$ symmetry.

The dilemma is easy to solve. In the multiplet under consideration we are dealing with two different levels of symmetry breaking: the $\mathrm{SU}(3)$ and the $\mathrm{SU}(2)$ ones. The former breaking does not split the masses within $S U(2)$ multiplets while the latter does. The $K_{2}^{* 0}$ is known to be modified by electromagnetic interaction. Therefore, the $K_{2}^{* \pm}$ should be recognized as better reflecting the properties of $\mathrm{SU}(3)$ broken multiplet. We thus define

$$
K_{2}^{*} \doteq K_{2}^{* \pm} \text {. }
$$

However, introducing a new definition for $K_{2}^{*}$ meson mass we are obliged to verify its agreement with the nonet criterion (18a). The MF, MOR and MM relations (which are defined for the nonet and decuplet masses) depend on the mass of $\mathrm{K}$ meson via the $\mathrm{b}$ parameter (3). In both the nonet and decuplet multiplets the meson $f_{2}(1270)$ is the $\mathrm{N}$-dominated $x_{N}$ isoscalar state and we can apparently see that its mass squared satisfies the inequality $x_{N}<a$. Then, keeping in mind MOR for the nonet (18a) and decuplet (23) and assuming the $f_{2}^{\prime}(1525)$ to be the S-dominated $x_{S}$ state we have:

if $x_{S}<b$ we are dealing with the nonet,

if $x_{S}>b$ we are dealing with the decuplet.

Using the data from tab.1 and definition (3) one can determine b. We should compare this value of $\mathrm{b}$ with the mass squared of the $f_{2}^{\prime}$ meson. For such a purpose we may neglect the errors of $K_{2}^{* \pm}(1430)$ and $a_{2}(1320)$ in calculating b as they are much smaller than the error of $f_{2}^{\prime}$ mass. We find

$$
b=2.3268 G e V^{2} .
$$

The nonet MOR (18a) requires

$$
f_{2}^{\prime}<b,
$$

while the decuplet MOR (23) imposes

$$
b<f_{2}^{\prime} .
$$

The mass of $f_{2}^{\prime}$ meson regarding experimental error is

$$
f_{2}^{\prime}=(2.326 \pm .015) G e V^{2} .
$$


Hence, within the error ranges of $f_{2}^{\prime}$ meson mass both the inequalities for the nonet (45) and for the decuplet (46) can be obeyed. The question posed in the title of this subsection is thus motivated but no answer is obtained. We must look for the answer in another way.

If the multiplet is a decuplet there should exist an extra meson which, together with the mesons (42), satisfies decuplet MOR restrictions (23). The decuplet MF (22) can be solved with respect to the mass of this extra meson. According to the nomenclature adopted for the isoscalar mesons (10) we denote this meson as $x_{2}$ (the symbols $x_{1}$ and $x_{3}$ are attributed to the mesons $f_{2}(1270)$ and $f_{2}^{\prime}(1525)$ dominated by $\mathrm{N}$ and $\mathrm{S}$ states). The solution is

$$
x_{2}=m_{2}^{2}=(1,327 G e V)^{2}
$$

and the mesons (42) are the components of decuplet if such a meson exists.

This solution is very sensitive to some of the input masses, especially to the mass of the mesons $K_{2}^{* \pm}$ and $f_{2}^{\prime}$. The mass (48) is calculated on the basis of mean experimental values cited in the table 1 . Only the mass of the meson $f_{2}^{\prime}$ is slightly changed: it is put $1526 \mathrm{MeV}$ instead of $1525 \mathrm{MeV}$.

The MM of this decuplet is

$$
V_{G}=\left[\begin{array}{ccc}
0.8123011 & 0.3290934 & 0.481523 \\
0.5692579 & -0.267674 & -0.777365 \\
-0.126934 & 0.90556510 & 0.404771
\end{array}\right] ;
$$

The mass of the pure G state is

$$
m_{G}=1350 \mathrm{MeV} .
$$

The signals with similar masses have been observed in several experiments during few decades. The bump is known as $f_{2}(1430)$ meson [14. The existence of this meson would support decuplet status of the mesons shown in the tab.1 and define it as G-dominated.

Note that this meson was looked for in early 80-th during exploring the misshapen nonet of $2^{++}$mesons [2, 4]. The failure of these attempts arise partly due to the wrongly chosen search area; the G-dominated meson was looked for above the mass of the meson $f_{2}^{\prime}(1525)$ instead below it. The mistake was a result of scanty data: at that time only one measurement has been recorded below this mass. There are now further measurements at our disposal but the existence of the particle still is not "firmly established" and needs confirmation. However, now we have more motivation for careful reanalysis.

Another question may also arise. If the G-dominated meson $f_{2}(1430)$ exists one may ask why? Room for it emerges due to anomalous electromagnetic split of the $K_{2}^{*}(1430)$ width. Is it an accident or testify some correlation? No trace of correlation between electromagnetic and strong interactions was seen so far.

\subsection{Quest for the second tensor multiplet}

Above the ground state $2^{++}$meson multiplet there exist further well established mesons and many signals waiting for confirmation. All these particles and signals are listed in the tab.2.

In the tab.2 we find only one isovector $a_{2}(1700)$ and one isospinor $K_{2}^{*}(1980)$ meson. This does not fit to ten isoscalar mesons seen in this energy region. But 
Table 2: Masses of tensor mesons observed above the ground state decuplet. Masses (in MeV) and confidence are quoted after [14]

\begin{tabular}{|c|c|c|c|c|c|}
\hline$f_{2}(1565)$ & $f_{2}(1640)$ & $a_{2}(1700)$ & $f_{2}(1810)$ & $f_{2}(1910)$ & $\bullet f_{2}(1950)$ \\
\hline $1562 \pm 13$ & $1639 \pm 6$ & $1732 \pm 16$ & $1815 \pm 12$ & $1903 \pm 9$ & $1944 \pm 12$ \\
\hline
\end{tabular}

\begin{tabular}{|c|c|c|c|c|c|}
\hline$K_{2}^{*}(1980)$ & $\bullet f_{2}(2010)$ & $f_{2}(2150)$ & $f_{J}(2220)$ & $\bullet f_{2}(2300)$ & $\left.\bullet f_{2}(2340)\right)$ \\
\hline $1973 \pm 33$ & $2011_{-76}^{+62}$ & $2157 \pm 12$ & $2231.1 \pm 3.5$ & $2297 \pm 28$ & $2339 \pm 55$ \\
\hline
\end{tabular}

the latter is poorly investigated and the situation may change in future. The content of the multiplet cannot be precisely determined, hence we focus our attention on selecting the particles to possible decuplet.

Only four out of the twelve signals listed in the tab.2, are "firmly established". Unfortunately, neither $a_{2}(1700)$ nor $K_{2}(1980)$ belong to this class. Yet we need definite values of their masses to put MOR delimitations of the decuplet. Therefore, we accept their identity numbers as the values of their masses:

$$
a=(1.700 \mathrm{GeV})^{2}, \quad b=2 K-a=(2.225 G e V)^{2} .
$$

The "firmly established" mesons constitute two pairs having so close mass values that their difference is smaller than the error. Such situation is exceptional in meson spectroscopy - usually the difference is bigger. Using the masses from tab. 2 we see that any mass of the pair of the "firmly established" mesons

$$
f_{2}(1950), \quad f_{2}(2010)
$$

satisfy the MOR restrictions on $x_{2}$ and any particle of the pair of "firmly established" mesons

$$
f_{2}(2300), \quad f_{2}(2340)
$$

satisfies the restriction on $x_{3}$. Besides, there are also two signals

$$
f_{2}(1565), \quad f_{2}(1640)
$$

satisfying the restriction on $x_{1}$.

Broad limits on variables $x_{i}$ and the freedom of the choice of the $a_{2}(1700)$ and $K_{2}^{*}(1980)$ masses would help to fit the MOR restrictions as well as the MF (22). Therefore, it is possible that the decuplet of these mesons exists. The mass of the corresponding G-dominated $2^{++}$meson is expected to be close to $2000 \mathrm{MeV}$.

At present, no tensor glueball candidate is promoted in this mass region but it was not always the case. Three out of four "firmly established" mesons listed in the tab.2 [14]

$$
f_{2}(2010), \quad f_{2}(2300), \quad f_{2}(2340)
$$

were discovered [17] in the eighties of the former century in the single experiment

$$
\pi^{-} p \longmapsto g_{T} n \longmapsto \Phi \Phi n .
$$

They were called $g_{T}$ mesons. Rich statistics of events justified the claim that the observed $\Phi \Phi$ signal descents from three separate mesons. 
The experiment raised big excitement since its authors claimed that the three mesons or "at least one of them" are glueballs. The belief was based on suggestion that $g_{T}$ mesons are created in the reaction which is doubly forbidden by OZI rule 3 .

Perhaps not all $g_{T}$ mesons are glueballs but if there exists any tensor glueball in this mass region then there should exist a decuplet as well. From two candidates for the role of $x_{2}$ shown in (52) we choose this one which is "firmly established". This points out the meson $f_{2}(2010)$ as G-dominated state. Unfortunately, there is no justification in support of similar choice between the mesons (53) and (54). Therefore, it is impossible to fix particle content of the decuplet. However, it is good luck that we can indicate candidate to the role of G-dominated isoscalar meson thus supporting the existence of the decuplet 4 .

\section{Summary}

The existence of the glueball implies enlarging the meson nonet to the decuplet where the glueball state $\mathrm{G}$ is mixed with the isoscalar $q \bar{q}$ nonet states. The discovery of the $G$ within the structure of decuplet helps in verification of its properties and makes the discovery more reliable. Therefore, decuplet occurs a right place for looking for it. The problem is in completing decuplet from the existing particles and describing it. We apply the VEC model. According to this model the multiplet is described by the master equations (ME). They form the specific sequence of equations depending only on the masses of the multiplet particles and the octet contents $l_{i}^{2}$ of the isoscalar physical states. The octet contents are the unknown variables of the sequence. Part of these equations (which is the basic system of the ME) determines the octet contents of the multiplet, the other ones define its mass formulae (MF). The ME explain the genesis of the MF and relate their number to the number of ME.

The number of ME is not given in advance as it is defined for each multiplet separately. To each number of ME there corresponds the individual multiplet of the physical particles. The multiplets having the same number of particles but different number of MF are considered as different. The existence of MF is not necessary for the multiplet existence.

The model well describes the nonets and predicts the existence of different kinds of them. The decuplets are defined just in the same way as other possible multiplets. The ME predict two kinds of decuplets. One of them has no MF, another has one MF. The latter is therefore more restrictive and its predictions

\footnotetext{
${ }^{3}$ The suggestion that the reaction (55) is doubly OZI-forbidden induced persistent opposition. When the dispute was prolonging excessively some people being interested in explaining the nature of the $g_{T}$ mesons but confused with this situation asked adversaries to work out a common conclusion at personal meeting. The appointment came face to face behind the closed doors. After this meeting an official statement was issued where the parties sustained their earlier positions 18. So impasse in interpretation of the reaction (51) was not overcome and interest to the nature of $g_{T}$ mesons gradually abated. However, the very existence of these mesons is not questioned and the masses measured in this experiment remain unchanged. Therefore, this event should not be forgotten. Possibly, now we shall have the opportunity to study structures of $g_{T}$ mesons without resolving the dispute on role of OZI rule in the reaction (56).

${ }^{4}$ One can wonder whether all $g_{T}$ mesons may belong to the same decuplet [19]. If it were so then there should exist also further tensor mesons $a_{2}$ and $K_{2}$ satisfying MOR. So far such mesons were not observed
} 
are more definite. It puts many constraints on the masses. Especially useful is the mass ordering rule (MOR) which is transparent and very effective in selecting the particles to the decuplet. The MOR joins conditions of the particle existence with the requirement of satisfying the MF.

The functions $l_{i}^{2}$, being solution of the basic sequence of ME, play important role in describing the multiplets.

1. They translate the natural positivity conditions $l_{i}^{2}>0(\mathrm{i}=1,2, .$.$) into the$ main restrictions on the masses of the nonets and decuplets.

2. They are the building blocks for constructing the mixing matrix as a function of masses showing at the same time room for ambiguities of its parameters.

The mixing matrix $V_{G}$ describes the decuplet of any domination pattern. It becomes completely determined if the "domination pattern" is indicated 5 .

The decuplet complying with the MF can be understood as a mixed state of the I-nonet and G. The admixture of $\mathrm{G}$ to the I-nonet transforms the pure (N, $\mathrm{S})$ nonet solution of the ME into the decuplet functions $x_{1}, x_{3}$ which, together with $x_{2}$, constitute the solution of ME for decuplet. The masses of the $x_{1}$ and $x_{3}$ mesons of decuplet do not differ much from the masses of parental I-nonet (N, $\mathrm{S})$. The mass gaps $\mathrm{N}-x_{1}$ and $x_{3}-\mathrm{S}$ are results of the $\mathrm{G}$ mixing. It is important that these gaps are smaller than the difference $b-a$ in all known multiplets. That indicates that the coupling of $\mathrm{G}$ to $(q \bar{q})$ states is relatively weak. This, in turn, assures us that I-nonet $(\mathrm{N}, \mathrm{S})$ - pattern of isoscalar mesons is preserved in the decuplet.

The standard domination pattern of a decuplet (40):

$$
x_{1} \sim N, \quad x_{2} \sim G, \quad x_{3} \sim S
$$

is attributed to the decuplet of any $J^{P C}$ having one MF. The flavor wave functions of these decuplets are determined via the G-shaped mixing matrix (33).

This procedure is applied for the description of possible tensor decuplets. We present two candidates.

One of the decuplets includes the mesons belonging to the currently known ground state nonet $2^{++}$and the isoscalar meson $f_{2}(1430)$. The nonet isoscalar state $f_{2}(1270)$ is $\mathrm{N}$-dominated and apparently its mass squared satisfies the relation $x_{N}<a$. Another isoscalar state $x_{S}$ which is S-dominated has the mass squared close to b. For a long time only this nonet was considered as the multiplet of the $2^{++}$mesons but now we find that the decuplet is also possible. This is due to the discovery of the anomalously large electromagnetic split of the $K_{2}^{*}(1430)$ mass. In this case the meson $f_{2}(1430)$ would serve as the lacking G-dominated state $x_{2}$. The candidate for the first tensor decuplet is

$$
a_{2}(1320), \quad K_{2}^{*}(1430), \quad f_{2}(1270), \quad f_{2}(1430), \quad f_{2}^{\prime}(1525) .
$$

The existence of the meson $f_{2}(1430)$ is uncertain. Its confirmation would disclose the G-dominated structure of this meson as well as existence of the decuplet. However, such correlation between effects of the strong and electromagnetic phenomena would perhaps need some explanation.

Constructing another decuplet candidate is an exercise of selecting the mesons

\footnotetext{
${ }^{5}$ fixing the decuplet domination pattern plays the role similar to the $(\mathrm{N}, \mathrm{S})$ attributing the isoscalar states of the I-nonet
} 
to the decuplet in the case of uncertain data. We propose

$$
a_{2}(1700), K_{2}(1980), f_{2}(1565) / f_{2}(1640), f_{2}(1950) / f_{2}(2010), f_{2}(2300) / f_{2}(2340)
$$

which includes mesons listed in the tab.2. This decuplet enters the field of the long-standing controversy about nature of $g_{T}$ mesons.

The G-dominated components of the tensor meson decuplets would be $f_{2}(1430)$ and $f_{2}\left((1950) / f_{2}(2010)\right.$.

We conclude with one more comment.

The VEC model describes meson multiplets of the broken $S U(3)$ flavor symmetry. The multiplets appear due to the difference between the masses of $\mathrm{K}$ and $\pi$ mesons (here we use pseudoscalar meson labels for any multiplet) and are treated in ME as an input. The difference between them is supposed to be an effect of the hard breaking of the $S U(3)$ symmetry. The mass splitting of the physical isoscalar decuplet mesons is regarded to be caused by other (soft) $S U(3)$ interaction between G, N, S states (the distinction of "very strong" and "middle strong" interaction was recognized from very beginning of flavor investigation). Soft interaction does not change the results of the hard one like big difference between the masses of $\mathrm{K}$ and $\pi$ mesons or determination of the domination pattern of the multiplet.

Nonet and decuplet may have the same $\mathrm{K}$ and $\pi$ masses and identifying the right multiplet may appear difficult if not all isoscalar mesons are seen. Then we should verify which of these multiplets better describes data.

\section{Conclusion}

The glueballs do exist. They can be found in the decuplets of mesons. If the decuplet obeys the mass formula the G-dominated state occupies central position between the isoscalar mesons $x_{i}$. The best candidates for glueball is $\eta(1405)$ and $f_{2}(1430)$ (if it exists). Their advantage is that they belong to decuplets gathering the states with the most precisely measured masses.

\section{Acknowledgments}

Author thanks Professor Anna Urbaniak-Kucharczyk - Dean of the Physical Department of the University of Lodz; Professor Paweł Maślanka and Professor Jakub Rembieliński - Chiefs of Theoretical Departments for their patient understanding and support; Professor S.B. Gerasimov and Professor V.A. Meshcheryakov (Dubna, Bogolyubov Laboratory of Theoretical Physics at Joint Institute of Nuclear Research) for durable friendly collaboration and any kind of help. Special thanks are expressed to Professor Piotr Kosiński for many interesting discussions, valuable comments and reading the manuscript; Dr Bartosz Zieliński help in computer operations is gratefully appreciated. 


\section{Appendix 1. The model of vanishing exotic commutators (VEC)}

The following sequence of exotic commutators is assumed to vanish

$$
\left[T_{a}, \frac{d^{j} T_{b}}{d t^{j}}\right]=0, \quad(j=1,2,3, \ldots)
$$

where $T$ is $S U(3)_{F}$ generator, $t$ is the time and $(a, b)$ is an exotic combination of indices, i.e. such that the operator $\left[T_{a}, T_{b}\right]$ does not belong to the octet representation. Substituting $\frac{d T}{d t}=i[H, T]$, and using the infinite momentum approximation for one-particle hamiltonian $H=\sqrt{m^{2}+p^{2}}$ we transform equations (59) into the system:

$$
\begin{aligned}
{\left[T_{a},\left[\hat{m^{2}}, T_{b}\right]\right] } & =0, \\
{\left[T_{a},\left[\hat{m^{2}},\left[\hat{m^{2}}, T_{b}\right]\right]\right] } & =0, \\
{\left[T_{a},\left[\hat{m^{2}},\left[\hat{m^{2}},\left[\hat{m^{2}}, T_{b}\right]\right]\right]\right] } & =0,
\end{aligned}
$$

where $\hat{m}^{2}$ is the squared-mass operator.

For the matrix elements of the commutators (60) between one-particle states (we assume one-particle initial, final and intermediate states) we obtain the sequence of equations involving expressions $\left\langle x_{8}\left|\left(m^{2}\right)^{j}\right| x_{8}\right\rangle$ with different powers $j=1,2,3, .$. , where $x_{8}$ is the isoscalar state belonging to the octet. Solving these equations, we obtain the sequence of formulae for a multiplet of the light mesons. We have

$$
\left\langle x_{8}\left|\left(\hat{m}^{2}\right)^{j}\right| x_{8}\right\rangle=\frac{1}{3} a^{j}+\frac{2}{3} b^{j} \quad(j=1,2,3, \ldots) .
$$

where $a$ is the mass squared of the isovector meson $a ; b$ is the mass squared of the subsidiary $s \bar{s}$ state,

$$
b=2 K-a,
$$

and $K$, in turn, is the mass squared of the isospinor $K$ meson.

The isoscalar octet state $\left|x_{8}\right\rangle$ can be represented as the linear combination of the physical isoscalar states

$$
\left|x_{8}\right\rangle=\sum l_{i}\left|x_{i}\right\rangle
$$

The coefficients $l_{1}, l_{2}, l_{3}, .$. determine octet contents of the physical isoscalar states $\left|x_{1}\right\rangle,\left|x_{2}\right\rangle,\left|x_{3}\right\rangle, \ldots$ Substituting (63) into (61) we obtain master equations (ME) of the multiplet:

$$
\sum l_{i}^{2} x_{i}^{j}=\frac{1}{3} a^{j}+\frac{2}{3} b^{j}, \quad(j=0,1,2,3, \ldots)
$$

where the $x_{1}, x_{2}, x_{3}, \ldots$ are isoscalar meson masses squared. Normalization condition of the $l_{i}$ coefficients is included into (64) as equation for $j=0$. 


\section{Appendix 2. Proof of MOR restrictions}

The MOR restrictions for the decuplet masses have been formulated long ago in several approaches e.g. [9, 11] but they are most apparently seen from fig.1 of 12 . We give now their simple algebraic proof.

The functions $l_{i}^{2}$ being the solution of the ME (8) have to satisfy conditions of the octet content positivity (12). Accepting the numbering of the isoscalar mesons (10) we fix the signs of the denominators of the functions (21). This determines the signs of their numerators:

$$
\begin{aligned}
& \left(x_{2}-a\right)\left(x_{3}-a\right)+2\left(x_{2}-b\right)\left(x_{3}-b\right)>0, \\
& \left(x_{1}-a\right)\left(x_{3}-a\right)+2\left(x_{1}-b\right)\left(x_{3}-b\right)<0, \\
& \left(x_{2}-a\right)\left(x_{1}-a\right)+2\left(x_{2}-b\right)\left(x_{1}-b\right)>0 .
\end{aligned}
$$

By combining the inequalities (65) with MF (22) we can obtain several restrictions on the masses of the decuplet particles.

In particular, assuming that $a>x_{1}$, i.e.

$$
\left(x_{1}-a\right)<0
$$

multiplying (65a) by (66) and then subtracting equation (22) we find, after omitting the positive factor $(b-a)$, the inequality

$$
\left(x_{2}-b\right)\left(x_{3}-b\right)<0 .
$$

Hence, according to (10)

$$
\left(x_{2}-b\right)<0, \quad\left(x_{3}-b\right)>0 .
$$

Repeating then the same operation with the product of (65c) and (68) we find

$$
\left(x_{1}-a\right)\left(x_{2}-a\right)<0
$$

and establish that

$$
\left(a-x_{2}\right)<0 .
$$

The collection of the inequalities (66), (70) and (68) form the rule (23).

The rule holds for any decuplet satisfying MF (22).

\section{References}

[1] H. Fritzsch, M. Gell-Mann, Proc. the XVI Int. Conf. on High Energy Physics, (12 Chicago-Batavia Ill.) 1972 vol. 2, p 135; H. Fritzsch, P. Minkowski, Nuovo Cim. A 30,393 (1975); A. de Rujula, H. Georgi, S.L. Glashow Phys. Rev. D12 147 (1975); J. F. Willemsen, Phys. Rev. D13 1327 (1976)

[2] J. L. Rosner, Phys. Rev. D24, 1347 (1981)

[3] P. G. O. Freund, Y. Nambu, Phys. Rev. Lett. 34, 1645 (1975); N. Fuchs, Phys. Rev. D 14, 1912 (1976); D. Robson, Nucl. Phys B130, 1977 (1977)

[4] J. L. Rosner, Phys. Rev. D 27, 1101 (1983); J. L. Rosner, S. F. Tuan, Phys. Rev. D 271544 (1983) 
[5] S. Meshkov, High Energy Physics, Plenum Press. New York (1985)

[6] F. Buisseret, V. Mathieu, C. Semay arXiiv: 0906.3098v2 [hep-ph];

P. Bicudo, S. Cotanch, F. J. Llanes-Estrada, D. G. Robertson Eur. Phys.J. C52 363 (2007) [arXiv:hep-ph/06021]

[7] C. J. Morningstar, M. Peardon, Phys. Rev.D60, 034509 (1999);

Y. Chen et al., Phys. Rev. D73, 014516 (2006)

[8] M. Majewski, W. Tybor, Acta Phys. Pol. B15 267 (1984)

[9] M. Majewski, W. Tybor, Acta Phys. Pol. B15, 777 (1984); Erratum, Acta Phys. Pol. B15, No 12 page 3 of the cover

[10] M. Majewski, Eur. Phys. J. C30, 223 (2003); hep-ph/0206285

[11] M. Majewski, Eur. Phys. J. C46 759 (2006); hep-ph/0509008

[12] M. Majewski, V. A. Meshcheryakov, J. Phys. G Nucl. Part. Phys 38 (2011) 035008;

[13] S. Godfrey and N. Isgur, Phys. Rev. D32 (1985) 189; L. Burakovsky and J. T. Goldman, Phys.Rev. D 57, 2879 (1998) hep-ph/9703271]; D. -M. Li, H. Yu and Q. -X. Shen, J. Phys. G 27 (2001) 807 [hep-ph/0010342]; C. -K. Chow and S. -J. Rey, JHEP9805 (1998) 010 hep-ph/9708355; F. Giacosa, T. Gutsche, V. E. Lyubovitskij, A. Faessler, Phys. Rev. D 72, 114021 (2005) hep-ph/0511171; Z. -C. Ye,X. Wang, X. Liu and Q. Zhao, Phys. Rev. D 86 (2012) 054025 [ arXiv:1206.0097 [hep-ph]]. F. Buisseret, V. Mathieu, C. Semay arXiv: 0906.3098v2 [hep-ph] (2009)

[14] Particle Data Group (Revew of Particle Properties (2014)) Chinese Physics C 38 N9 (2014) 090001

[15] C. Amsler, T. Gutsche, S. Spanier, N. A. Tornqvist, ibid p 630

[16] C. Amsler, G. L. Masoni. ibid p 680

[17] A. Etkin et al., Phys. Rev. Let.40 422 (1978); 491620 (1982); A. Etkin et al.,Phys. Lett B 165217 (1685); Phys. Lett B 201568 (1988)

[18] H. J. Lipkin, Phys. Lett. B 124509 (1983); S. J. Lindenbaum, Phys, Lett. B 131221 (1983); S. J. Lindenbaum, H.J. Lipkin, Phys. Lett. B 149407 (1984)

[19] B. Kozlowicz, M. Majewski, Acta Phys. Pol. B20 869 (1989) 